ORIGINAL ARTICLE

\title{
Chronic behavioral stress exaggerates motor deficit and neuroinflammation in the MPTP mouse model of Parkinson's disease
}

\author{
E Lauretti ${ }^{1}$, A Di Meco ${ }^{1}$, S Merali $^{2}$ and D Praticò ${ }^{1}$
}

Environmental stressor exposure is associated with a variety of age-related diseases including neurodegeneration. Although the initial events of sporadic Parkinson's disease (PD) are not known, consistent evidence supports the hypothesis that the disease results from the combined effect of genetic and environmental risk factors. Among them, behavioral stress has been shown to cause damage and neuronal loss in different areas of the brain, however, its effect on the dopaminergic system and PD pathogenesis remains to be characterized. The $\mathrm{C} 57 \mathrm{BL} / 6$ mice underwent chronic restraint/isolation (RI) stress and were then treated with 1-methyl-4-phenyl-1,2,3,6-tetrahydropyridine (MPTP), whereas the control mice were treated only with MPTP and the effect on the PD-like phenotype was evaluated. The mice that underwent RI before the administration of MPTP manifested an exaggerated motor deficit and impairment in the acquisition of motor skills, which were associated with a greater loss of neuronal tyrosine hydroxylase and astrocytes activation. By showing that RI influences the onset and progression of the PD-like phenotype, our study underlines the novel pathogenetic role that chronic behavioral stressor has in the disease process by triggering neuroinflammation and degeneration of the nigral dopaminergic system.

Translational Psychiatry (2016) 6, e733; doi:10.1038/tp.2016.1; published online 9 February 2016

\section{INTRODUCTION}

Parkinson's disease (PD) is an irreversible age-associated chronic neurodegenerative condition characterized by the loss of dopaminergic neurons located in the pars compacta of the substantia nigra (SN) that is located in the midbrain and projects to the striatum and other subcortical regions. The resulting loss of dopamine leads to rigidity, resting tremor and postural disabilities. ${ }^{1}$ The precise etiology of PD has been under investigation for more than a century. Although rare genetically linked cases of early-onset PD have been reported, most incidences are late-onset and sporadic in nature, and thought to result from the combined effects of genetic and environmental risk factors. ${ }^{2}$

As an environmental risk factor, chronic stress has been suggested to have a relevant role in the development of neurodegeneration. ${ }^{3}$ Several studies have widely investigated the effect that chronic stress, dysregulation of stress hormone levels, such as glucocorticoids, and stress-related disorders have on different areas of the brain. Thus, permanent loss of hippocampal neurons, atrophy of the hippocampus as well as alteration of synaptic plasticity are all well-known consequences of previous exposure to stress. ${ }^{3,4}$ Therefore, it has been suggested that acute and chronic stress may either trigger an earlier onset or worsen the development of PD neuropathology.

Although emerging evidence suggests that exposure to chronic stress can affect the normal dopamine (DA) system activity in the central nervous system, especially in the mesolimbic and mesocortical pathways, this interaction may be more complex than expected and dependent on the type and the length of the exposure. In fact, although some studies report that mice exposed to acute stress manifest an increase of the DA release in the mesocorticolimbic system, a reduced dopaminergic activity has been shown in the nucleus accumbens after exposure to a forced swimming task. ${ }^{4,5}$ Interestingly, although a previous paper reported that immobilization stress can increase DA levels that can then cause oxidative damages in the nigrostriatal system, the effect that this type of stressor has on the 1-methyl-4-phenyl1,2,3,6-tetrahydropyridine (MPTP) model of PD has not yet been investigated. ${ }^{6}$

To this end, in the current study, we examined the behavioral and neuropathological consequences that pre-exposure to chronic restraint/isolation (RI) has on the PD-like phenotype development and progression in C57BL/6 mice after being treated with MPTP. ${ }^{7}$

Under our experimental conditions, we observed that compared with naive mice, the group undergoing RI before receiving the MPTP showed a more severe motor deficit and a reduction in the ability to acquire proper motor skills, which were associated with a more severe depletion of dopaminergic neurons in the SN region, and higher levels of glial fibrillary acidic protein (GFAP), a marker of astrocytic cells activation.

\section{MATERIALS AND METHODS}

Animals and MPTP treatment

All animal procedures were approved by the Institutional Animal Care and Usage Committee and were in accordance with the National Institute of Health guidelines. Adult male and female C57BL/6 mice were used in this study. Mice were kept in a pathogen-free environment, on a 12-h light/

\footnotetext{
'Department of Pharmacology and Center for Translational Medicine, School of Medicine, Temple University, Philadelphia, PA, USA and ${ }^{2}$ Department of Pharmaceutical Sciences, School of Pharmacy, Temple University, Philadelphia, PA, USA. Correspondence: Professor D Praticò, Department of Pharmacology and Center for Translational Medicine, School of Medicine, Temple University, 947 Medical Education and Research Building, 3500 North Broad Street, Philadelphia, PA 19140, USA.

E-mail: praticod@temple.edu

Received 29 June 2015; revised 25 August 2015; accepted 19 September 2015
} 
dark cycle and had access to food and water ad libitum. The study involved a total of 40 mice that were randomized into four groups: CTR $(n=10), \mathrm{Rl}$ $(n=9)$, MPTP $(n=12)$ and MPTP/RI $(n=9)$. Starting at 7 months of age, two groups, RI and MPTP/RI, underwent restraint isolation for 31 days before receiving vehicle and MPTP, respectively. MPTP $\left(25 \mathrm{mg} \mathrm{kg}^{-1}\right.$ in saline, intraperitoneally) and vehicle were administrated once a day for 7 consecutive days. At 8 months of age, all the mice were tested in the behavioral tasks described below and subsequently killed. At the time of killing, the animals were perfused with ice-cold $0.9 \%$ phosphate-buffered saline containing ethylene-diamine-tetra-acetic acid $\left(2 \mathrm{mmol} \mathrm{I}^{-1}\right), \mathrm{pH}$ 7.4. The brains were removed and immediately dissected in two halves. One half was stored at $-80^{\circ} \mathrm{C}$ for biochemistry assays, the other half was fixed in $4 \%$ paraformaldehyde, pH 7.4 for immunohistochemistry studies. All the tests and analyses were always performed by an investigator who was blind to the treatment and genotype.

\section{Restraint stress procedure}

The chronic Rl stress protocol was carried out as previously described. ${ }^{8}$ Briefly, each mouse was placed in the restrainer device $(1.5 \times 4 \mathrm{~cm}$, Braintree Scientific, Braintree, MA, USA) for $6 \mathrm{~h}$ per day for 31 consecutive days. The devices had multiple air holes and allowed animals to stretch their legs, but not to move within the restrainers. Immediately after this period, each animal was returned to the home cage and had access to food and water ad libitum.

\section{Rotarod test}

A Rotarod instrument with automatic timers and falling sensors (Omnitech Electronics, Columbus, OH, USA) was used. Rotarod testing was performed on 4 consecutive days. The first test was performed $2.5 \mathrm{~h}$ after the last MPTP injection. The mice were placed individually on a $30 \mathrm{~mm}$ diameter rotating cylinder suspended above a cage floor. The length of time the mice managed to remain on the rod was automatically recorded. The mice underwent six trials per day and the maximal observation time for each trial was $90 \mathrm{~s}$. During the training phase (day 1-3), the speed of the rotation was increased gradually from 0 to 15 r.p.m. during the first $15 \mathrm{~s}$ and held constant at that rate for the rest of the trial $(75 \mathrm{~s})$. During the test (day 4), the speed of rotation was accelerated gradually from 0 to 90 r.p.m. during the $90 \mathrm{~s}$ of the trial.

\section{Biochemical analyses}

The mice were killed 4 days after completion of the Rotarod testing. The striatal contents of DA and its major metabolite 3,4-dihydroxyphenylacetic acid (DOPAC) were measured by high-performance liquid chromatography analysis, as previously described. ${ }^{9}$ Briefly, the striata were rapidly dissected, sonicated and mixed with $75 \mu \mathrm{l}$ of borate buffer. Twenty microliters of the AccQ-Fluor reagent ( $N$-hydroxysuccinimidyl-6-aminoquinoyl carbamate) was then added to give a final volume of $100 \mu$, the samples vortexed and then spiked with $10 \mu \mathrm{l}$ of $1 \mathrm{~mm}$ aqueous solutions of DA and DOPAC. The Waters high-performance liquid chromatography used included a quaternary pump (model 625), a system controller (model 600E), an autosampler (model 715) and a fluorescence detector (model 470). Waters Millenium software (Milford, MA, USA) was used to control the system, collect data and calculate peak area values. A $5-\mu \mathrm{m}$ silica particle C8 Symmetry column $(3.9 \times 150 \mathrm{~mm}$, inner diameter) with a $100-\AA ̊$ pore size (Waters) was used for separation. The elution buffer consisted of $0.14 \mathrm{M}$ sodium acetate, $0.017 \mathrm{~m}$ triethylamine- $\mathrm{HCl}, \mathrm{pH} 5.05$ (eluent A). Fluorescence excitation was at $250 \mathrm{~nm}$, and emission was detected at $395 \mathrm{~nm}$. Elution was at $1.0 \mathrm{ml} \mathrm{min}$. The mobile phase gradient was as previously described for polyamine analysis, ${ }^{10}$ except the last step was a linear gradient starting at $83 \%$ eluent A, $17 \%$ acetonitrile extending to $60 \%$ eluent A, 40\% acetonitrile over $20 \mathrm{~min}$. All the samples were analyzed within $1 \mathrm{~h}$ of preparation, and each sample was always analyzed in triplicate.

\section{Immunoblotting}

The mouse striata were extracted in RIPA buffer as previously described. ${ }^{11}$ Briefly, total protein concentration was measured with BCA Protein Assay Kit (Pierce, Rockford, IL, USA). The samples were separated on SDS polyacrylamide gel electrophoresis by using a 10\% Bis-Tris gel and then transferred onto nitro-cellulose membranes (Bio-Rad, Richmond, CA, USA), blocked with Odyssey blocking buffer for $1 \mathrm{~h}$ at room temperature and incubated with primary antibodies overnight at $4{ }^{\circ} \mathrm{C}$. After three washing cycles, membranes were incubated with IRDye $800 \mathrm{CW}$ labeled secondary antibody (LI-COR Bioscience, Lincoln, NE, USA) for $1 \mathrm{~h}$ at room temperature and developed with Odyssey Infrared Imaging System (LI-COR Bioscience). The following primary antibodies were used: tyrosine hydroxylase $(\mathrm{TH}$; 1:100; catalog \#AB152, Millipore, Billerica, MA, USA), GFAP (1:200; catalog \#sc-33673, Santa Cruz, Dallas, TX, USA), D2R (1:20; catalog \#sc-5303, Santa Cruz), D1R (1:20; catalog \# sc-1434, Santa Cruz). Actin was always used as an internal loading control.

\section{Immunohistochemistry}

Immunostaining was performed as previously described. ${ }^{12}$ Briefly, serial coronal brain sections were cut throughout each brain and mounted on 3-aminopropyltriethoxysilane-coated slides. The sections were deparaffinized, hydrated, rinsed with phosphate-buffered saline and pretreated with citric acid $(10 \mathrm{~mm})$ for 5 min for antigen retrieval, then with $3 \% \mathrm{H}_{2} \mathrm{O}_{2}$ in methanol for $30 \mathrm{~min}$ to eliminate endogenous peroxidase activity and with blocking solution (2\% normal serum in Tris buffer, $\mathrm{pH} 7.6$ ). Subsequently, the sections were incubated overnight at $4{ }^{\circ} \mathrm{C}$ with the primary antibodies GFAP (1:200; Santa Cruz) and TH (1:100; Millipore), then with secondary antibody and developed using the avidin-biotin complex method (Vector Laboratories, Burlingame, CA, USA) with 3,3-diaminobenzidine as chromogen. Consecutives sections were incubated in the absence of primary antibodies to ensure specificity of staining.

\section{Statistical analyses}

All the data are expressed as mean+s.e.m. The one-way analysis of variance test, Bonferroni multiple-comparison test and the two-tailed Student's $t$ test were performed using Prism 5.0 (Graph Pad Software, La Jolla, CA, USA) to determine the statistical significance, with significance set at $P<0.5$.

\section{RESULTS}

Restraint impairs motor coordination and learning skills

To investigate the impact of chronic RI stress on the PD-like phenotype secondary to MPTP in C57BL/6 mice, first we examined their motor skills on the Rotarod. ${ }^{13}$ Compared with control naive mice, the ones undergoing Rl did not show any significant differences in their general locomotor activity as both groups of mice spent comparable time on the rod over the 4 days of testing (Figure 1). By contrast, the locomotor activity was drastically reduced in MPTP and MPTP+RI groups compared with RI alone and control mice. Furthermore, the exposure to RI before the MPTP led to more severe motor deficits and impairments in the acquisition of Rotarod motor skill, as unlike the MPTP+RI group, mice receiving the MPTP alone spent increasingly more time on the rod over the 3 days of training (Figure 1). No gender differences were observed in the performance of the Rotarod test when males and females were analyzed separately.

\section{Influence of RI on MPTP-dependent reduction of DA in the striatum}

As it is well known that MPTP toxicity is responsible for a profound loss of dopaminergic neurons in the striatum, next we assayed the content of DA and DOPAC in the different experimental groups. As shown in Figure 2a, we noticed that compared with controls, the mice exposed to Rl alone did not have any significant changes in the levels of DA and DOPC, but, as expected, MPTP treatment resulted in a significant reduction in the striatum levels of DA and DOPAC. However, no significant differences for both compounds were observed between MPTP and MPTP+RI (Figure 2a). In addition, among the different groups, no differences between males and females were observed in the striatal content of DA and DOPAC.

\section{$\mathrm{Rl}$ increases the expression of D2DR}

As previous work showed that exposure to stress can modulate levels of the two major DA receptors (that is, D1DR and D2DR), 
DAY 1

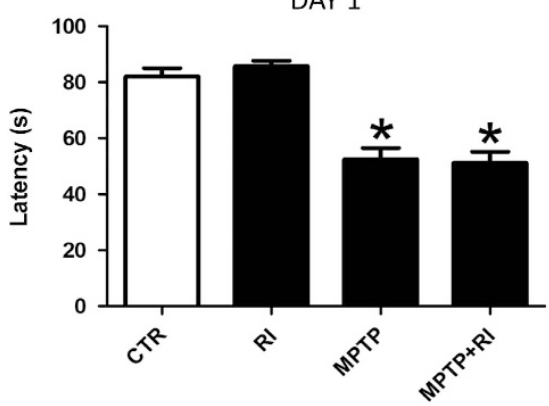

DAY 3

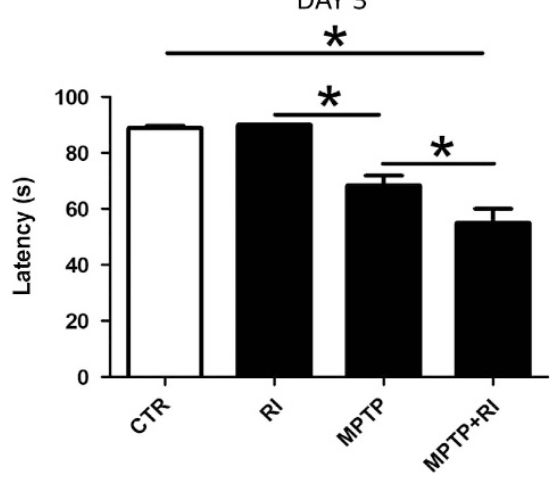

DAY 2

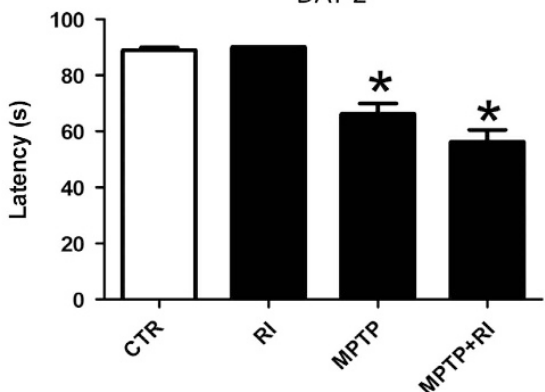

DAY 4

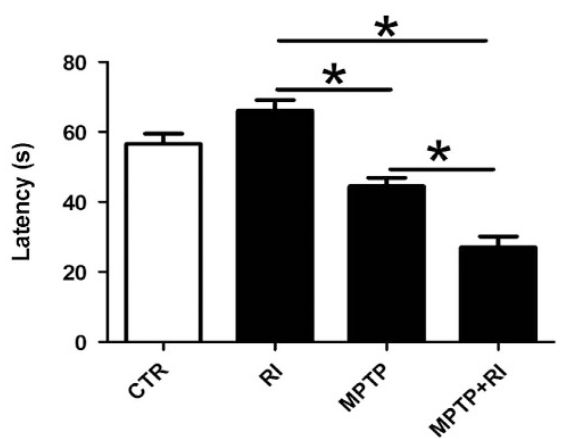

Figure 1. Restraint stress worsens MPTP-dependent motor deficits and impairs motor skill learning ability. Days 1-3: Latency to fall off the Rotarod of C57BL/6 mice receiving vehicle (CTR, $n=10)$; MPTP $(n=12)$; MPTP+RI $(n=9)$; RI $(n=9)$. Mice underwent a training phase consisting of six trials per day, for 3 days. In this phase, the speed of the rotation was increased gradually from 0 to 15 r.p.m. during the first 15 s, and then held constant at that rate for the rest of the trial $\left.{ }^{*} P>0.05\right)$. Day 4: Rotarod performance of the same groups of mice on the test day after the 3 days of training. The speed of rotation was accelerated gradually from 0 to 90 r.p.m. for the 90 s of the trial $\left(^{*} P<0.05\right)$. Results are mean \pm s.e. m. MPTP, 1-methyl-4-phenyl-1,2,3,6-tetrahydropyridine; Rl, restraint/isolation.

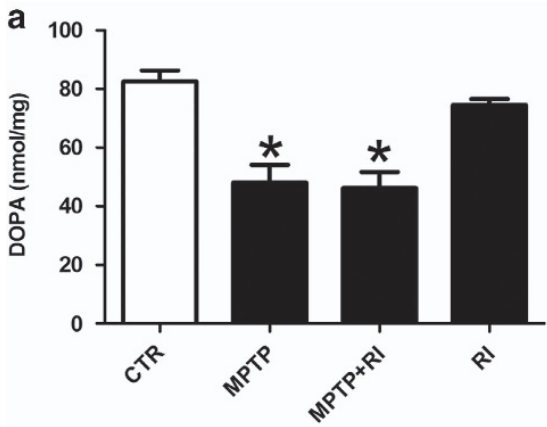

b
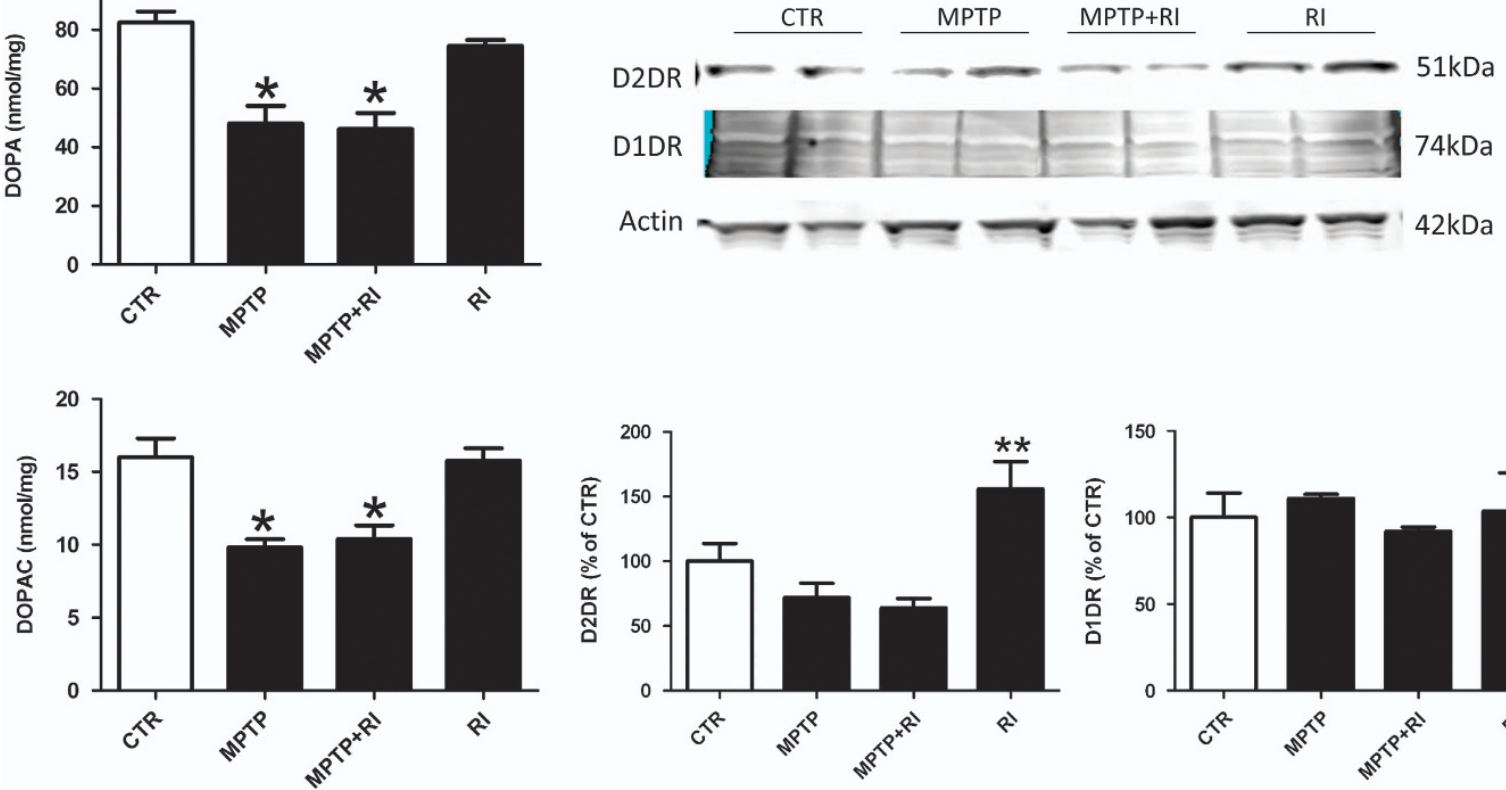

figure 2. Changes in DA system after restraint stress and MPTP treatment. (a) Level of DA and its metabolite DOPAC in striatal homogenates of the four groups of C57BL/6 mice receiving vehicle (CTR, $n=10)$; MPTP $(n=12)$; MPTP+RI $(n=9)$; and RI $(n=9)\left({ }^{*} P<0.05\right)$. (b) Representative western blot analysis of striatal levels of DA receptors D1DR and D2DR in the same groups of mice and densitometric analyses of their immunoreactivities $\left({ }^{*} P<0.05\right)$. Results are mean \pm s.e.m. DA, dopamine; DOPAC, 3,4-dihydroxyphenylacetic acid; MPTP, 1-methyl-4-phenyl1,2,3,6-tetrahydropyridine; RI, restraint/isolation. ${ }^{* *} P<0.001$. 
a

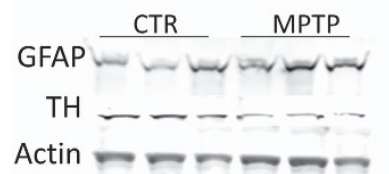

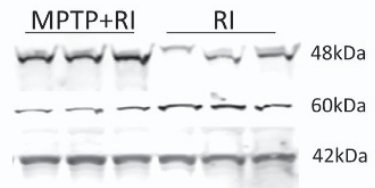
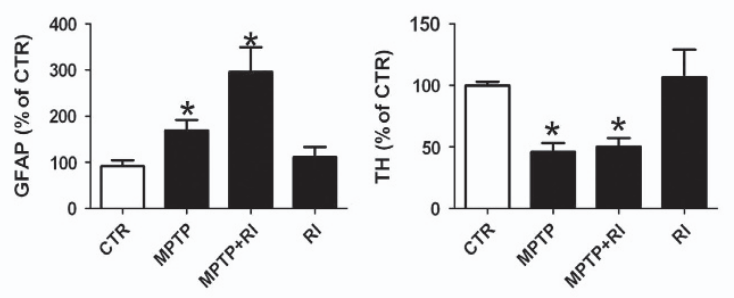

b

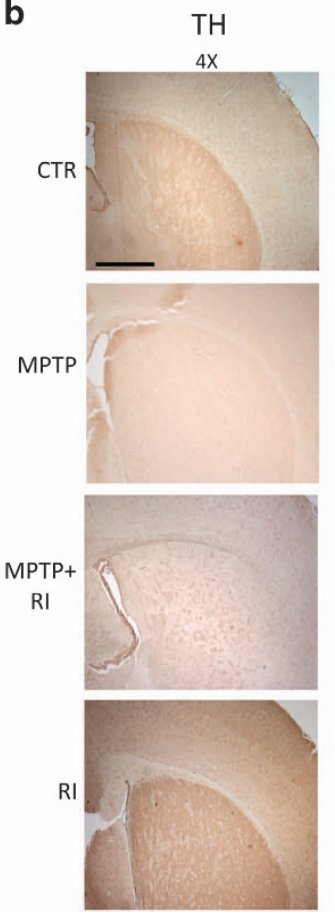

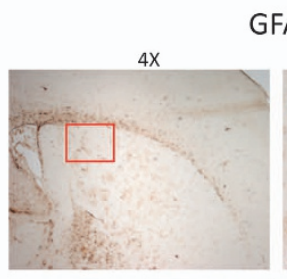

GFAP
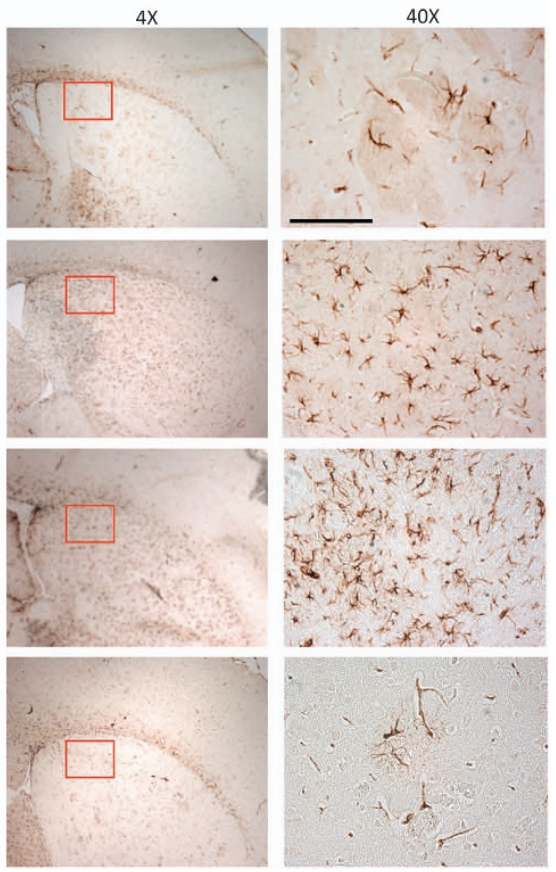

Figure 3. Effect of restraint on the MPTP-dependent striatal cell loss and astrocytes activation. (a) Representative western blot and relative densitometric analyses of TH and GFAP immunoreactivity in striatum homogenates of the four groups of C57BL/6 mice receiving vehicle (CTR, $n=10)$; MPTP ( $25 \mathrm{mg} \mathrm{kg}^{-1}$ in saline intraperitoneally for a week; $\left.n=12\right)$; MPTP+RI $(n=9)$; and RI $\left(n=9 ;{ }^{*} P<0.05\right)$. Results are mean \pm s.e.m. (b) Representative images of immunohistochemistry analyses for TH and GFAP reactivity in the striatum region of the same groups of mice (scale bars, $20 \mu \mathrm{m}(\times 4) ; 100 \mu \mathrm{m}(\times 40))$. GFAP, glial fibrillary acidic protein; MPTP, 1-methyl-4-phenyl-1,2,3,6-tetrahydropyridine; Rl, restraint/isolation; $\mathrm{TH}$, tyrosine hydroxylase.

a

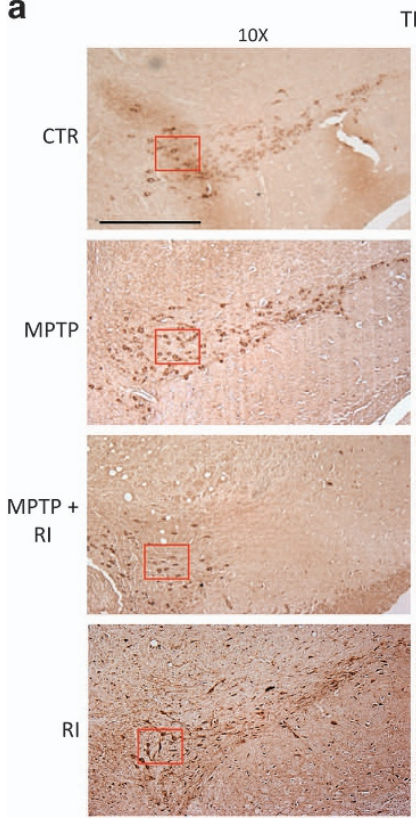

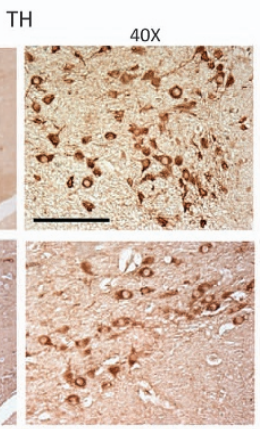
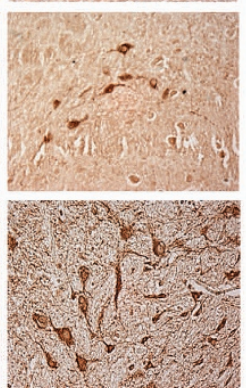

b

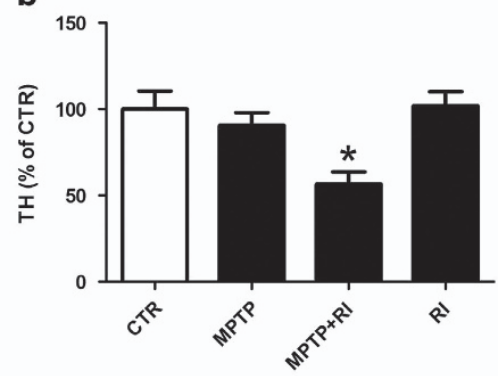

Figure 4. Chronic behavioral stress exaggerates the MPTP-dependent cell loss in the substantia nigra. (a) Representative images of immunohistochemistry analyses for TH reactivity in the substantia nigra of C57BL/6 mice receiving vehicle alone (CTR, $n=10)$; MPTP $(n=12)$; $\operatorname{MPTP}+\mathrm{RI}(n=9)$; or RI $(n=9$; scale bars, $100 \mu \mathrm{m}(\times 10) ; 100 \mu \mathrm{m}(\times 40))$. (b) Quantitative analysis of the TH immunoreactivity shown in a $\left({ }^{*} P<0.05\right)$. Results are mean \pm s.e.m. MPTP, 1-methyl-4-phenyl-1,2,3,6-tetrahydropyridine; Rl, restraint/isolation; TH, tyrosine hydroxylase.

next we investigated whether their protein levels were different as a result of the treatments. As shown in Figure $2 b$, we did not find significant changes in D2DR between the MPTP and $M P T P+R I$ groups. However, consistently with previous findings, the RI mice showed a significant increase in the striatal level of D2DR when compared with controls. ${ }^{14}$ No significant differences in the steady-state levels of D1DR were detected among the four groups. 
a

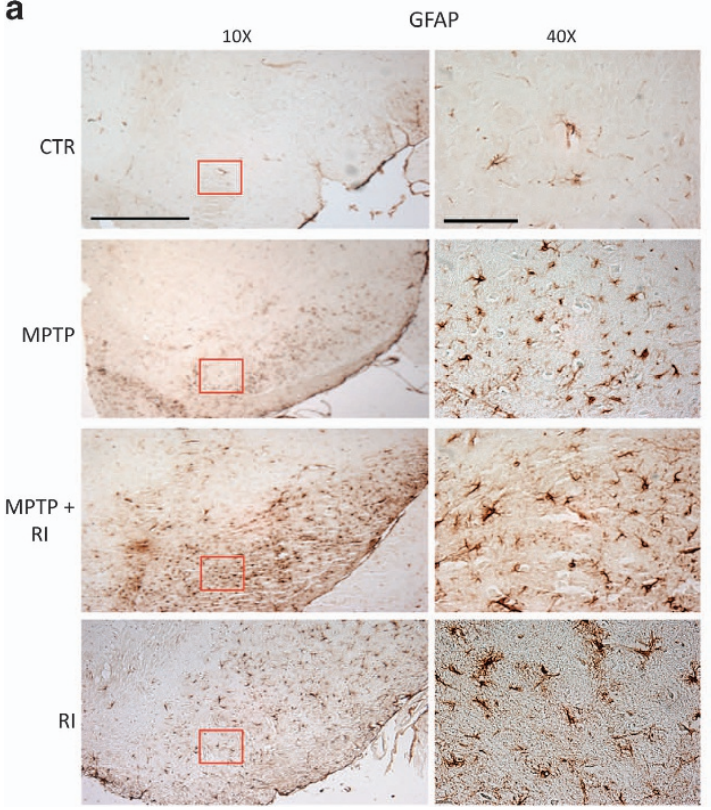

b

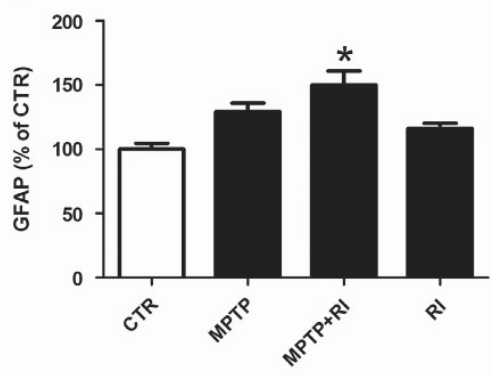

Figure 5. Chronic behavioral stress worsens the MPTP-dependent astrocytosis in the substantia nigra. (a) Representative images of immunohistochemistry analyses for GFAP reactivity in the substantia nigra of C57BI/6 mice receiving vehicle alone $(C T R, n=10)$; MPTP $(n=12)$; MPTP+RI $(n=9)$; or RI $(n=9$; scale bars, $100 \mu \mathrm{m}(\times 10)$; $100 \mu \mathrm{m}(\times 40))$. (b) Quantitative analysis of the GFAP immunoreactivity shown in a $\left({ }^{*} P<0.05\right)$. Results are mean \pm s.e.m. GFAP, glial fibrillary acidic protein; MPTP, 1-methyl-4-phenyl-1,2,3,6-tetrahydropyridine; Rl, restraint/ isolation.

\section{RI results in greater cell loss in SN after MPTP}

To verify whether our RI paradigm had an effect on the MPTPinduced neuronal degeneration, next we assayed brain levels of the principal enzyme involved in DA synthesis, the TH enzyme. ${ }^{15}$ As shown in Figure 3a, compared with controls we did not observe any significant changes in the striatal level of $\mathrm{TH}$ in the brain of RI mice. By contrast, TH levels were significantly lower in MPTP and $\mathrm{MPTP}+\mathrm{RI}$ mice when compared with controls, but there was no significant difference between these two groups (Figure 3a). This observation was further corroborated by immunohistochemistry analysis of the same striatal region (Figure $3 \mathrm{~b}$ ).

Interestingly, when we looked at $\mathrm{TH}$ in the $\mathrm{SN}$ of all the groups, MPTP+RI mice showed a lower number of TH-positive cells in this area compared with mice receiving MPTP alone. Conversely, RI animals displayed no decrease in the density of TH-positive cells in the SN area when compared with naive mice (Figures $4 a$ and b).

RI enhances GFAP immunoreactivity in the striatum and SN Compared with control mice, the one receiving MPTP had significant increase in the level of GFAP, a marker for astrocytes activation, in the striatum region (Figure 3a). Furthermore, MPTP $+\mathrm{RI}$ mice showed a higher increase in this marker when compared with the MPTP group, but no changes were observed in the group of mice undergoing the RI paradigm alone (Figure 3a). These results for the different groups were confirmed by immunohistochemistry analyses of the same brain region (Figure $3 \mathrm{~b}$ ).

Interestingly, when we looked at the SN of the same groups, we observed that the immunoreactive areas for GFAP had a trend toward an increase for both the MPTP and RI groups, which reached statistical significance only in the MPTP+RI mice group (Figures $5 \mathrm{a}$ and $\mathrm{b}$ ).

\section{DISCUSSION}

The present study shows that exposure to chronic behavioral stress results in the worsening of MPTP-induced motor deficits, exacerbation of DA depletion and neuroinflammation in the C57BL/6 mice.

Consistent evidence has widely shown that the DA system is very susceptible to stress, as studies in both human and rats indicated that stress and stress hormones can affect the normal function of the motor system. ${ }^{16}$ Accordingly, it has been suggested that acute or chronic stress might lead to augmented neuronal degeneration and concomitant cognitive deficits by worsening the PD pathology. ${ }^{16}$ However, despite a previous study suggested that stress can contribute to the oxidative damage in the nigrostriatal system, ${ }^{6}$ the effect of direct chronic stress exposure on the MPTP mouse model has not been investigated yet.

Thus, the purpose of this study was to examine the behavioral and neuropathological effects of chronic behavioral stress in this mouse model of PD. Our results show that, the pre-exposure to RI before the actual treatment with the neurotoxic agent MPTP exacerbates the onset and development of the PD-like phenotype. Interestingly, we observed that mice undergoing the chronic behavioral stress also manifested impairment in the acquisition of the motor skills since they lacked the ability to learn them during the training session of the Rotarod task. Owing to the extensive training and the low rotation speed, we can exclude that this outcome depended on gait disability. Instead, considering the importance of the nigrostriatal projection to the cerebral cortex and striatum for the learning aspect of the Rotarod task, we hypothesize that chronic behavioral stress might affect locomotor skills as well as cognitive and learning function.

In search for biochemical substrates for the observed behavioral outcomes, we investigated whether and how the DA system of these mice was affected by the different treatments. First, assessment of striatal content for DA and DOPAC showed that compared with vehicle controls, the MPTP and MPTP+RI groups had a significant reduction for both. However, mice receiving the $\mathrm{RI}$ alone did not have any changes in the levels of these metabolites in the striatum. A similar pattern was observed when in the same region, we analyzed the steady-state levels of $\mathrm{TH}$, the rate-limiting enzyme for the formation of DA. By contrast, 
immunohistochemistry analyses of the SN of the different groups showed a statistically significant reduction in the immunoreactivity for TH only in the MPTP+RI group, but not in the MPTP or RI groups.

The physiological actions of DA are mediated by a group of G-protein-coupled receptors commonly divided into two major classes: D1 (D1DR and D5DR) and D2 (D2DR, D3D4 and D4DR). ${ }^{17}$ Specifically, the effect of DA on locomotor activity is regulated by D1DR and D2DR receptors. D1DR receptors are found exclusively on postsynaptic neurons and have a moderate stimulatory effect; in contrast, D2DR receptors are found both pre- and post synapse and their effect on locomotor activity is more complex than the D1 receptors. ${ }^{17}$ Under our experimental conditions, we found a significant increase in D2DR receptors in the RI mice compared with control, but no changes in the other groups. Given that a recent study has described how RI affects the D2DR receptors density in the striatum, the results are not surprising. ${ }^{14}$

The loss of DA neurons seen in PD patients and in the MPTP model is known to be typically associated with an intense glial response. $^{18}$ In fact, under pathological conditions, activated microglia and astrocytes release pro-inflammatory cytokines such as IL-1 $\alpha, I L-1 \beta$ and IL- 6 , and TNF- $\alpha$ that are considered detrimental to $\mathrm{SN}$ dopaminergic neurons. ${ }^{19}$ For this reason, we assayed the expression levels of GFAP, a marker for astrocytes activation, in the striatum and SN of all the experimental groups. Thus, compared with vehicle control, we first observed that MPTP treatment resulted in a significant increase in the steady-state levels of GFAP, and that this increase was further and significantly exacerbated in the MPTP group pretreated with RI in both the brain regions.

In summary, our study demonstrates that exposure to chronic behavioral stress, as produced by daily Rl, by inducing a proinflammatory state exaggerates the motor deficit and the MPTPinduced cell loss in the SN, which leads to an impairment in the ability to acquire motor skills.

We conclude that chronic behavioral stress should be considered as having an important active role in the onset and development of the PD phenotype. Correction of this environmental factor could be a potential and viable preventative therapeutic strategy for individuals who are exposed to it.

\section{CONFLICT OF INTEREST}

The authors declare no conflict of interest.

\section{ACKNOWLEDGMENTS}

This study was supported, in part, by the Wanda Simone Endowment for Neuroscience (DP).

\section{REFERENCES}

1 Shiotsuki H, Yoshimi K, Shimo Y, Funayama M, Takamatsu Y, Ikeda K et al. A rotarod test for evaluation of motor skill learning. J Neurosci Methods 2010; 189: 180-185.

2 Speciale SG. MPTP: insights into parkinsonian neurodegeneration. Neurotoxicol Teratol 2002; 24: 607-620.

3 Kibel A, Drenjancević-Perić I. Impact of glucocorticoids and chronic stress on progression of Parkinson's disease. Med Hypotheses 2008; 71: 952-956.

4 Gil M, Armario A. Chronic immobilization stress appears to increase the role of dopamine in the control of active behavior in the forced swimming test. Behav Brain Res 1998; 91: 91-97.

5 Sedelis M, Schwarting RK, Huston JP. Behavioral phenotyping of the MPTP mouse model of Parkinson's disease. Behav Brain Res 2001; 125: 109-125.

6 Kim ST, Choi JH, Chang JW, Kim SW, Hwang O. Immobilization stress causes increases in tetrahydrobiopterin, dopamine, and neuromelanin and oxidative damage in the nigrostriatal system. J Neurochem 2005; 95: 89-98.

7 Tieu K. A guide to neurotoxic animal models of Parkinson's disease. Cold Spring Harb Perspect Med 2011; 1: a009316.

8 Joshi YB, Giannopoulos PF, Chu J, Sperow M, Kirby LG, Abood ME et al. Absence of ALOX5 gene prevents stress-induced memory deficits, synaptic dysfunction and tauopathy in a mouse model of Alzheimer's disease. Hum Mol Genet 2014; 23: 6894-6902.

9 Coppi A, Merali S, Eichinger D. The enteric parasite Entamoeba uses an autocrine catecholamine system during differentiation into the infectious cyst stage. J Biol Chem 2002; 277: 8083-8090.

10 Merali S, Clarkson AB. Polyamine analysis using $N$-hydroxysuccinimidyl-6-aminoquinoyl carbamate for pre-column derivatization. J Chromatogr B Biomed Sci Appl 1996; 675: 321-326.

11 Di Meco A, Joshi YB, Praticò D. Sleep deprivation impairs memory, tau metabolism, and synaptic integrity of a mouse model of Alzheimer's disease with plaques and tangles. Neurobiol Aging 2014; 35: 1813-1820.

12 Lauretti E, Di Meco A, Chu J, Praticò D. Modulation of AD neuropathology and memory impairments by the isoprostane F2a is mediated by the thromboxane receptor. Neurobiol Aging 2014; 36: 812-820.

13 Brooks SP, Dunnett SB. Tests to assess motor phenotype in mice: a user's guide. Nat Rev Neurosci 2009; 10: 519-529.

14 Lim MM, Xu J, Holtzman DM, Mach RH. Sleep deprivation differentially affects dopamine receptor subtypes in mouse striatum. Neuroreport 2011; 22: 489-493.

15 Haavik J, Toska K. Tyrosine hydroxylase and Parkinson's disease. Mol Neurobiol 1998; 16: 285-309.

16 Smith LK, Jadavji NM, Colwell KL, Katrina Perehudoff S, Metz GA. Stress accelerates neural degeneration and exaggerates motor symptoms in a rat model of Parkinson's disease. Eur J Neurosci 2008; 27: 2133-2146.

17 Beaulieu JM, Gainetdinov RR. The physiology, signaling, and pharmacology of dopamine receptors. Pharmacol Rev 2011; 63: 182-217.

18 Smeyne RJ, Jackson-Lewis V. The MPTP model of Parkinson's disease. Brain Res Mol Brain Res 2005; 134: 57-66.

19 Rappold PM, Tieu K. Astrocytes and therapeutics for Parkinson's disease. Neurotherapeutics 2010; 7: 413-423.

(c) (i) $९$ This work is licensed under a Creative Commons Attribution-

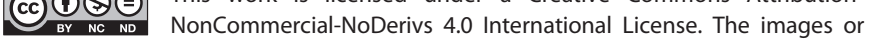
other third party material in this article are included in the article's Creative Commons license, unless indicated otherwise in the credit line; if the material is not included under the Creative Commons license, users will need to obtain permission from the license holder to reproduce the material. To view a copy of this license, visit http:// creativecommons.org/licenses/by-nc-nd/4.0/ 